\title{
Guanabenz Acetate Induces Endoplasmic Reticulum Stress-Related Cell Death in Hepatocellular Carcinoma Cells
}

Hyo Jeong Kang ${ }^{1 *}$

Hyang Sook Seol ${ }^{2 *}$. Sang Eun Lee ${ }^{2}$ Young-Ah Suh ${ }^{2} \cdot$ Jihun Kim ${ }^{1}$

Se Jin Jang ${ }^{1,2} \cdot$ Eunsil $\mathrm{Yu}^{1,3}$

${ }^{1}$ Department of Pathology, ${ }^{2}$ Asan Institute for Life Science, ${ }^{3}$ Asan Liver Center, Asan Medical Center, University of Ulsan College of Medicine, Seoul, Korea

Received: January 5, 2018

Revised: December 28, 2018

Accepted: January 14, 2019

\section{Corresponding Author}

Se Jin Jang, MD, PhD

Department of Pathology, Asan Medical Center, University of Ulsan College of Medicine,

88 Olympic-ro 43-gil, Songpa-gu, Seoul 05505,

Korea

Tel: +82-2-3010-5608

Fax: +82-2-472-7898

E-mail: jangsejin@amc.seoul.kr

Eunsil Yu, MD, PhD

Department of Pathology, Asan Medical Center, University of Ulsan College of Medicine, 88 Olympic-ro 43-gil, Songpa-gu, Seoul 05505 Korea

Tel: +82-2-3010-4552

Fax: +82-2-472-7898

E-mail: d890075@gmail.com

*Hyo Jeong Kang and Hyang Sook Seol contributed equally to this work.
Background: Development of chemotherapeutics for the treatment of advanced hepatocellular carcinoma ( $\mathrm{HCC}$ ) has been lagging. Screening of candidate therapeutic agents by using patientderived preclinical models may facilitate drug discovery for HCC patients. Methods: Four primary cultured HCC cells from surgically resected tumor tissues and six HCC cell lines were used for high-throughput screening of 252 drugs from the Prestwick Chemical Library. The efficacy and mechanisms of action of the candidate anti-cancer drug were analyzed via cell viability, cell cycle assays, and western blotting. Results: Guanabenz acetate, which has been used as an antihypertensive drug, was screened as a candidate anti-cancer agent for HCC through a drug sensitivity assay by using the primary cultured HCC cells and HCC cell lines. Guanabenz acetate reduced HCC cell viability through apoptosis and autophagy. This occurred via inhibition of growth arrest and DNA damage-inducible protein 34, increased phosphorylation of eukaryotic initiation factor $2 \alpha$, increased activating transcription factor 4, and cell cycle arrest. Conclusions: Guanabenz acetate induces endoplasmic reticulum stress-related cell death in HCC and may be repositioned as an anti-cancer therapeutic agent for HCC patients.

Key Words: Hepatocellular carcinoma; Primary cell culture; Drug sensitivity; Drug repositioning; Guanabenz acetate
Hepatocellular carcinoma (HCC) is one of the most common malignancies worldwide, and its incidence is increasing because of the dissemination of hepatitis B and C viruses. ${ }^{1,2} \mathrm{HCC}$ is the third most common cause of cancer-related deaths, with approximately 600,000 deaths per year. ${ }^{3-6}$ Although surgical resection and transplantation are effective treatments for HCC, most patients present with advanced-stage disease at the time of diagnosis and are not suitable candidates for curative surgery. ${ }^{4}$ Inoperable HCC patients undergo various loco-regional treatments, including transarterial chemoembolization, radiofrequency ablation, and percutaneous ethanol injection, with or without systemic chemo- therapy using adriamycin, cisplatin, or sorafenib. ${ }^{7-9}$ Despite the development of various treatments for advanced HCC patients, HCC remains difficult to treat because these methods do not significantly improve mortality. ${ }^{10}$ Therefore, novel approaches to develop targeted therapeutic agents are needed.

Immortalized cancer cell lines have been typically used for highthroughput screening of chemical libraries to develop novel therapeutics in the past. Although immortalized cancer cell lines have the advantage of being robust and tractable, they have significant limitations recapitulating tumor heterogeneity of in vivo human tumors. ${ }^{11-13}$ Primary cultured cancer cells from patient tumors 
have been reported to maintain similarity with the original tumors with respect to histopathology, biomarker expression, genomic mutation profiles, and drug responsiveness. ${ }^{14}$ Therefore, primary cultured cells have been suggested as a substitute preclinical model of various tumors for screening and evaluation of anti-cancer therapeutic candidates.

The traditional approach to drug discovery involves de novo identification and validation of new molecular entities, which is a time-consuming and costly process. ${ }^{15}$ Despite huge investments in drug discovery and development, and explosive advances in biological technologies during the past decades, the number of new drugs introduced into the clinic has not increased accordingly. Alternatively, identification of new therapeutic indications for approved drugs i.e., drug repurposing has attracted particular attention for anti-cancer drug discovery. ${ }^{15-17}$ This strategy has several advantages over the traditional de novo drug discovery approach, including reducing development time, costs, and the risks associated with pharmacokinetics and toxicology. ${ }^{17-20}$ For drug repurposing research, several established clinical drug libraries approved by the U.S. Food and Drug Administration (FDA), European Medicines Agency (EMA), and other agencies are available.

In the present study, we conducted drug repurposing study and could select guanabenz acetate (GA), an antihypertensive drug, as a candidate therapeutic molecule by high throughput screening on patient derived primary cultured HCC cells using the Prestwick Chemical Library which are all approved by the U.S. FDA and EMA. Mechanistically, GA inhibited growth arrest and DNA damage-inducible protein 34 (GADD34)-mediated dephosphorylation of eukaryotic initiation factor $2 \alpha$ (eIF2 $\alpha$ ), and subsequently induced apoptosis and autophagy of the HCC cells.

\section{MATERIALS AND METHODS}

\section{Ethical approval}

This study was approved by the Institutional Review Board of Asan Medical Center with a waiver of informed consent (IRB No. 2012-0112).

\section{Patients and samples}

Four patients with HCC, who were confirmed to have the disease via liver protocol dynamic computed tomography scans at Asan Medical Center, Seoul, Korea, were selected to establish primary cultured cells. A portion of the tumor tissues obtained from surgical resection were fixed in cold $2 \%$ formaldehyde for 4 hours and embedded in paraffin at $56^{\circ} \mathrm{C}$. Sections from the paraffin blocks ( $4-\mu \mathrm{m}$ thick) were stained with hematoxylin and eosin (H\&E). All H\&E slides were reviewed by two pathologists (E.Y. and H.J.K.) who were blinded to the clinical information.

\section{Primary culture of HCC cells}

The fresh tissues of the four cases obtained during surgery were put into a tube containing Dulbecco's modified Eagle medium: nutrient mixture F-12 (DMEM/F12; Sigma-Aldrich, St. Louis, MO, USA), with penicillin and streptomycin, and were transported to the tissue culture room. After removing normal liver tissue and connective tissue, tumor tissues were minced with scissors and subsequently digested with $0.1 \%$ type IV collagenase (Sigma-Aldrich) in a shaking incubator for 60 minutes at $37^{\circ} \mathrm{C}$. After incubation, tissues were washed three to four times with DMEM/F12 containing 10\% fetal bovine serum (FBS; SigmaAldrich). The HCC pellets obtained after centrifugation were plated on collagen type I dishes and incubated at $37^{\circ} \mathrm{C}$ in a $5 \%$ $\mathrm{CO}_{2}$ atmosphere. To favor the adhesion and growth of epithelial tumor cells, hepatocyte basal media containing human epidermal growth factor, transferrin, hydrocortisone, bovine serum albumin, ascorbic acid, GA-1000 (gentamicin and amphotericin B), insulin, 10\% FBS, and a Triiodothyronine-SingleQuots kit (Biocompare, South San Francisco, CA, USA) were used to culture primary HCC tumor cells. The cultured HCC cells were harvested and stored in liquid nitrogen.

\section{MTT cell proliferation assay/drug sensitivity assay}

Two hundred and fifty-two small molecules from the Prestwick Chemical Library (Prestwick Chemical, Illkirch, France) were blindly selected for screening of anti-cancer effect. The primary cultured HCC cells $\left(10^{3}\right.$ cells/well) were seeded in collagen type I coated 96-well plates. Following a 24-hour incubation at $37^{\circ} \mathrm{C}$, the cells were treated with 252 small molecules $(20 \mu \mathrm{M}$ each). After 72 hours, $50 \mathrm{mg} / \mathrm{mL}$ of MTT [3-(4,5-dimethylthiazol-2-yl)-2,5-diphenyltetrazolium bromide] reagent were added to each well, and the plates were incubated for 4 hours at $37^{\circ} \mathrm{C}$. After the reduced formazan precipitate was dissolved in dimethyl sulfoxide, the absorbance at $540 \mathrm{~nm}$ was measured using a microplate reader (EL800, BioTek, Winooski, VT, USA). The concentration of candidate molecules to inhibit $50 \%$ of cell viability ( $\mathrm{IC}_{50}$ value) was calculated using CalcuSyn Software (Biosoft, Cambridge, UK). These results were validated in six stable HCC cell lines (i.e., SNU398, SNU423, SNU449, SNU475, Hep3B, and Huh7). Five HCC cell lines (i.e., SNU398, SNU423, SNU 449, SNU475, and Huh7) and one HCC cell line (Hep3B) were respectively grown in RPMI 1640 medium (Sigma-Aldrich) and Minimum Essential Medium (Sigma-Aldrich), both supplemented 
with 10\% FBS, penicillin, and streptomycin.

\section{Small interfering RNA transfection}

Hep3B and Huh7 HCC cells were treated with small interfering RNA (siRNA) specific to GADD34 and non-specific siRNA (Genolution Pharmaceuticals Inc., Seoul, Korea). Targeting sequences of siRNA were as follows: siGADD34-1, 5'-GGAUAAGGAAGAUGAUUCAUU-3'; siGADD34-2, 5'-GCCUAUAAUUUAUUAACUAUU-3'; and as a negative control, non-specific siRNA, 5'-CCUCGUGCCGUUCCAUCAGGUAGUU-3'. HCC cells were transiently transfected with GADD34 siRNA or non-specific siRNA with Lipofectamine 2000 (Invitrogen, Carlsbad, CA, USA). Six hours later, the medium was replaced with regular culture medium, and the cells were incubated. The efficiency of silencing was assessed via western blotting 48 hours after transfection.

\section{Western blot analysis}

Harvested cells were lysed in Cell Lysis Buffer (Cell Signaling Technology, Beverly, MA, USA) for 20 minutes and centrifuged at 13,500 rpm for 5 minutes. Twenty micrograms of proteins were separated by sodium dodecyl sulfate-polyacrylamide gel electrophoresis using 8\%-12\% gels and transferred to nitrocellulose membranes (Potran nitrocellulose membrane, Whatman, Kent, UK) using an iBlot dry blotting system (Invitrogen). The membranes were blocked with 5\% non-fat dried milk and incubated with specific primary antibodies for (1) GADD34 (1:1,000), activating transcription factor 4 (ATF4; 1:1 000), p53 (1:1,000), and $\beta$-actin $(1: 2,000)$ from Santa Cruz Biotechnology (Dallas, TX, USA); (2) eIF2 (1:1,000), phospho-eIF2 (p-eIF2, 1:1,000), p-p53 (Ser15, 1:2,000), p21 Waf1/Cip1 (1:500), cyclin B1 (1:1,000), cyclin E2 (1:1,000), p-cdc2 (p-cdk1, 1:2,000), Bax $(1: 1,000)$, Bcl-xL (1:2,000), procaspase-9 (1:2,000), and cleaved caspase-9 (1:1,000) from Cell Signaling Technology. After incubation with appropriate horseradish peroxidase-conjugated secondary $\operatorname{IgG}$ antibodies, bands were detected using ECL reagent (Amersham-GE Healthcare Life Sciences, Pittsburgh, PA, USA).

\section{Cell cycle assay}

The effect of the agent on the cell cycle of HCC cell lines was analyzed by flow cytometry using propidium iodide (PI) staining. Briefly, $10^{6}$ cells were seeded in $60-\mathrm{mm}$ dishes and incubated with the agent $(30 \mu \mathrm{M})$ or phosphate-buffered saline (PBS) as a control for 24 hours at $37^{\circ} \mathrm{C}$. After incubation, the cells were fixed with ice-cold $70 \%$ ethanol at $4^{\circ} \mathrm{C}$ overnight, washed twice with PBS, incubated with $10 \mu \mathrm{g} / \mathrm{mL}$ RNase A, and stained with $30 \mu \mathrm{g} / \mathrm{mL}$ PI. The stained cells were subsequently analyzed using flow cytometry (FACSCalibur machine, Becton Dickinson, Mountain View, CA, USA) with a 560-nm dichroic mirror and a 600 -nm pass filter (bandwidth, $35 \mu \mathrm{m}$ ). The percentages of cells in G1, S, and G2 phases were determined using Cell Quest 3.1 software (Becton Dickinson).

\section{RESULTS}

\section{GA screened as a potential therapeutic drug for HCC}

Four primary cultured HCC cells were established using surgically resected fresh tumor tissues from four HCC patients and subsequently confirmed via pathologic examination (Fig. 1A, B). The clinicopathologic features of the patients are listed in Table 1.
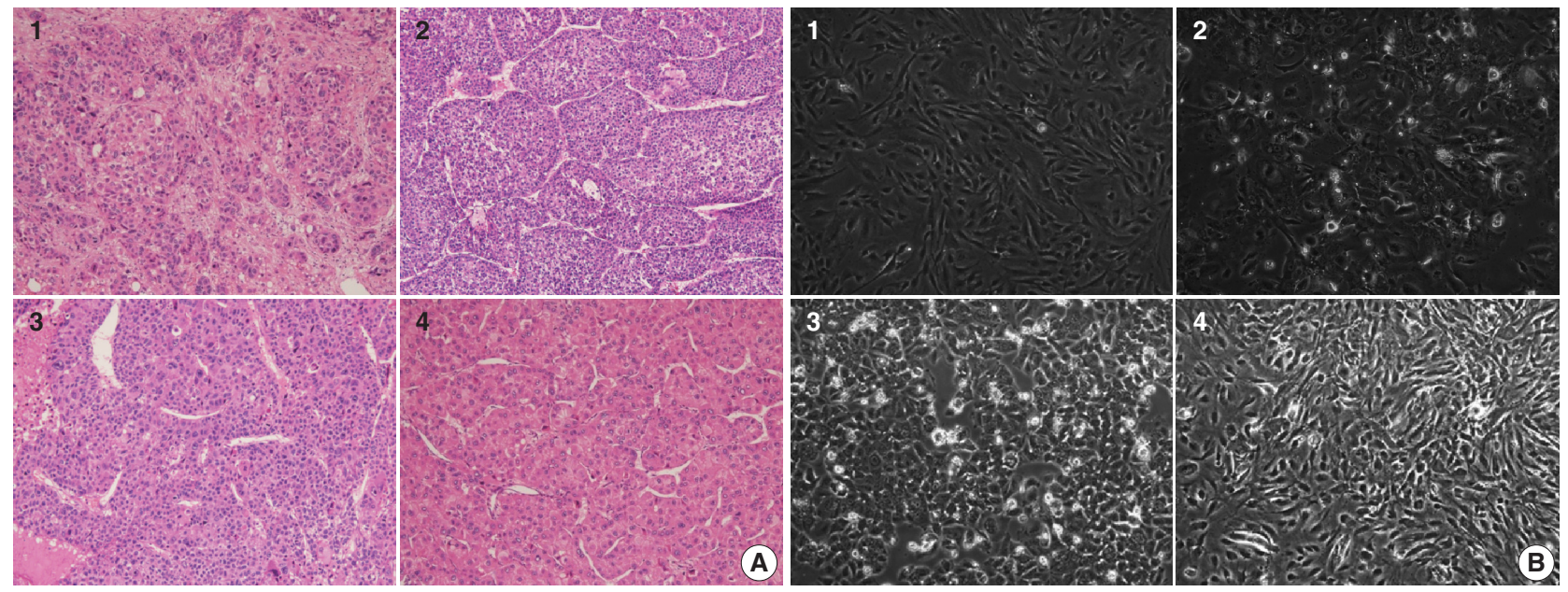

Fig. 1. (A) Histological features of surgically resected hepatocellular carcinomas, cases 1-4, respectively. (B) Cytological characteristics of primary cultured hepatocellular carcinoma cells with 3 passages, cases 1-4, respectively. 
Table 1. Clinicopathologic features of four cases of hepatocellular carcinoma from which the PDX model was established

\begin{tabular}{lcccccc}
\hline Case No. & Age $(\mathrm{yr})$ & Sex & Size $(\mathrm{cm})$ & Edmondson-Steiner grade & Underlying disease & Previous treatment \\
\hline 1 & 58 & $\mathrm{M}$ & 13 & III & Cirrhosis/HBV & No \\
2 & 56 & $\mathrm{M}$ & 4.5 & III & Periportal fibrosis/HBV & No \\
3 & 70 & $\mathrm{M}$ & 4.7 & III & Cirrhosis/HBV & TACE \\
4 & 55 & $\mathrm{M}$ & 11 & II & Cirrhosis/HBV & No \\
\hline
\end{tabular}

PDX, patient derived xenograft; HBV, hepatitis B virus; TACE, transarterial chemoembolization.

High-throughput drug screening using the Prestwick Chemical Library, which contains 1,120 FDA-approved drugs (Supplementary Table S1), was performed on the four primary cultured HCC cells. Two hundred and fifty-two of the 1,120 molecules were randomly selected and screened and identified GA as a candidate. The results were confirmed using individual in vitro assay. After 72-hour incubation with a concentration range of the molecules $(0-50 \mu \mathrm{M})$, cell viability was inhibited more than $50 \%$ with 20 $\mu \mathrm{M}$ GA (i.e., compound 41) (Fig. 2A) in three out of four primary cultured HCC cells, measured using the MTT assay.

\section{GA inhibited growth of $\mathrm{HCC}$ cell lines}

To further validate the anti-cancer effect of GA in HCC, six HCC cell lines were treated with GA (Sigma-Aldrich) or with a control molecule, sorafenib. After 72-hour incubation with GA, growth of Hep3B $\left(\mathrm{IC}_{50}=30 \mu \mathrm{M}\right)$ and $\operatorname{Huh} 7\left(\mathrm{IC}_{50}=50 \mu \mathrm{M}\right)$ were inhibited (Fig. 2B). The $\mathrm{IC}_{50}$ values of GA for SNU423, SNU449, and SNU475 cells were $100 \mu \mathrm{M}$, and the viability of SNU 398 cells did not drop below $50 \%$, even at $100 \mu \mathrm{M}$ GA (Fig. 2B). When the inhibitory effects of sorafenib on cell proliferation were compared with those of GA, the decline in viability occurred at similar or lower concentrations with sorafenib in all six HCC cell lines (Fig. 2B).

\section{GA targets PERK signaling pathway through GADD34 inactivation}

GA is an agonist of the $\alpha 2$ adrenergic receptor that is used as an antihypertensive drug. GA is also known to block dephosphorylation of eIF2 $\alpha$ by inactivation of GADD3 4 in the protein kinase RNA-like ER kinase (PERK) signaling pathway, which would finally lead to expression of genes involved in apoptosis and autophagy. Therefore, the protein expression levels of genes associated with PERK signaling were evaluated by western blotting in the Hep3B and Huh7 cell lines to study the mechanisms of GA in HCC. The level of p-eIF2 $\alpha$ was upregulated, and ATF4 protein was increased after GA treatment of Hep3B and Huh7 cells (Fig. 3A). In addition, when HCC cells were transfected with GADD3 4 siRNA, levels of p-eIF2 $\alpha$ and ATF4 were upregulated in both Hep3B and Huh7 HCC cells compared with levels in control HCC cells (Fig. 3A). These results reveal that GA acts as an inhibitor of dephosphorylation of eIF2 $\alpha$, likely by inactivation of GADD34 in HCC.

\section{GA induces apoptosis and autophagy-related cell death in HCC cells}

ATF4, a transcription factor that induces the expression of genes, is involved in amino acid responses, metabolism, antioxidant responses, apoptosis, autophagy, and GADD34-mediated effects. To assess if GA induces HCC cell death via apoptosis and/ or autophagy following ATF4 upregulation, expression levels of apoptosis-related proteins (i.e., Bax, Bcl-xL, procaspase-9, and cleaved caspase-9) and an autophagy-related protein (i.e., microtubule-associated protein light chain 3, LC3) were examined in Hep3B and Huh7 by western blotting. In the Hep3B and Huh7 cells treated with GA, Bax protein expression was higher, while $\mathrm{Bcl}-\mathrm{xL}$ protein expression was lower than the expressions in control HCC cells (Fig. 3B). In addition, protein levels of procaspase-9 were decreased, while cleaved caspase- 9 was increased in both Hep3B and Huh7 cells (Fig. 3C). Both LC3-I and LC3-II protein levels increased in Hep3B, but only LC3-II protein level increased in Huh7 treated with GA (Fig. 3D). These results clearly demonstrate that both mechanism of apoptosis and autophagy play a role in GA-induced cell death. Activated ATF4 in the PERK pathway is considered an essential gateway for apoptosis and autophagy in HCC cells.

\section{Different types of cell cycle arrest in different HCC cell lines treated with GA}

To examine whether GA reduces HCC cell viability by inducing cell cycle arrest, flow cytometry was performed. The expression levels of checkpoint proteins in the G1/S and G2/M transitions and cell cycle regulating factors were then examined by western blotting. The cell cycle distribution pattern was different between the two HCC cell lines. In Hep3B cells treated with GA, the percentage of $\mathrm{G1}$ phase cells increased, while the percentages of $\mathrm{S}$ and G2 phase cells concomitantly decreased (Fig. 4A). In contrast to Hep3B, the cell cycle distribution of Huh7 treated with GA revealed an increase in the percentage of G2 phase cells, while 

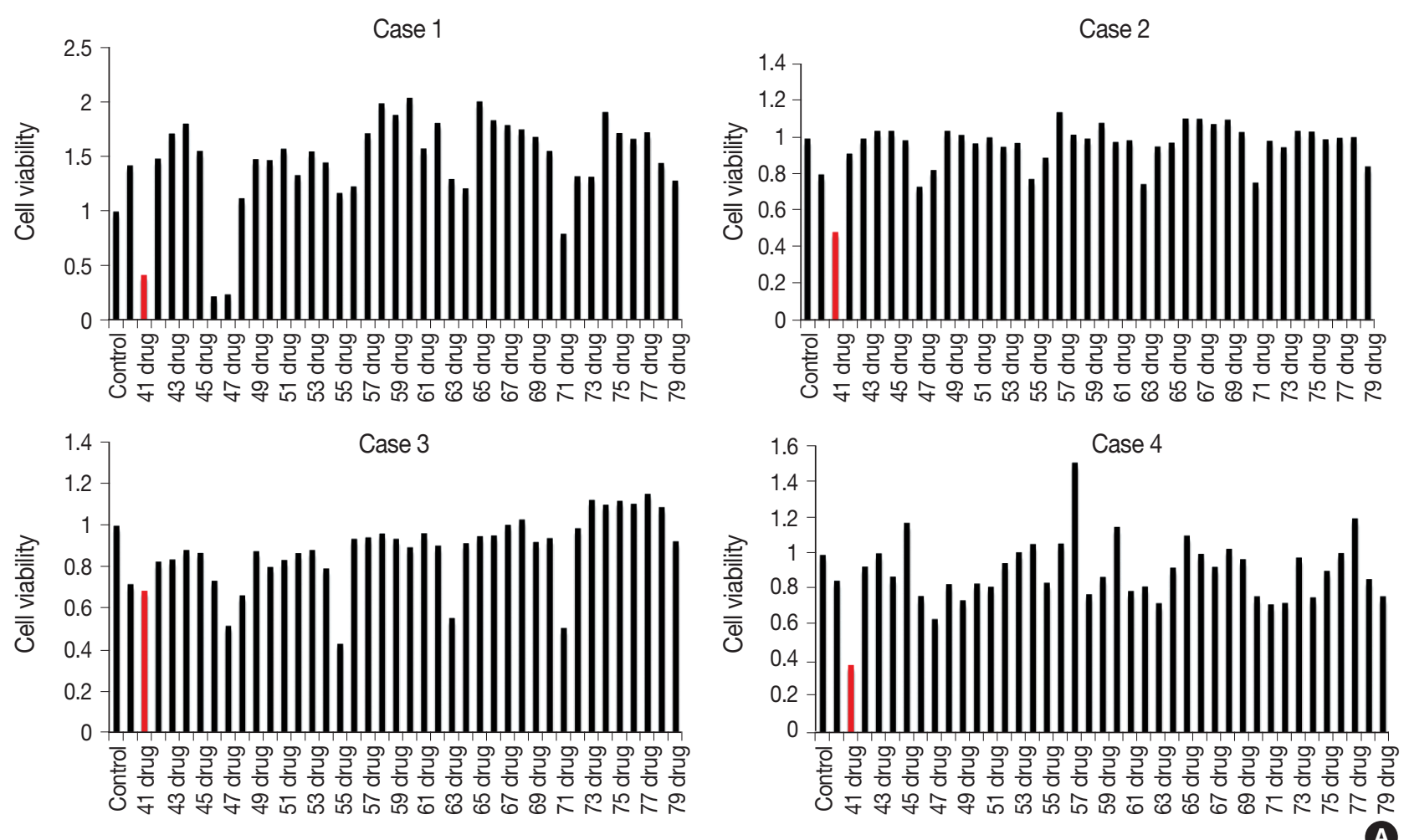

Hep3B

Huh7

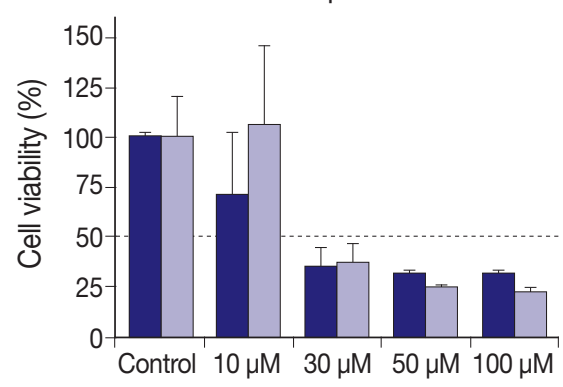

SNU449

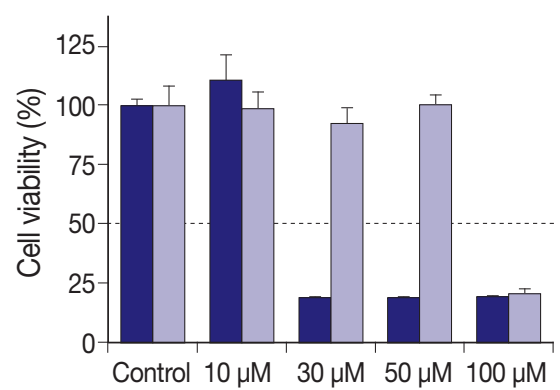

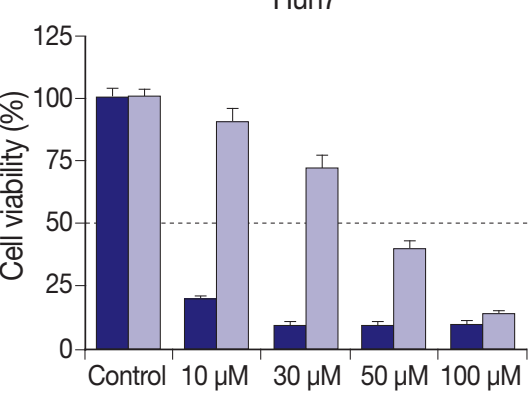

SNU475

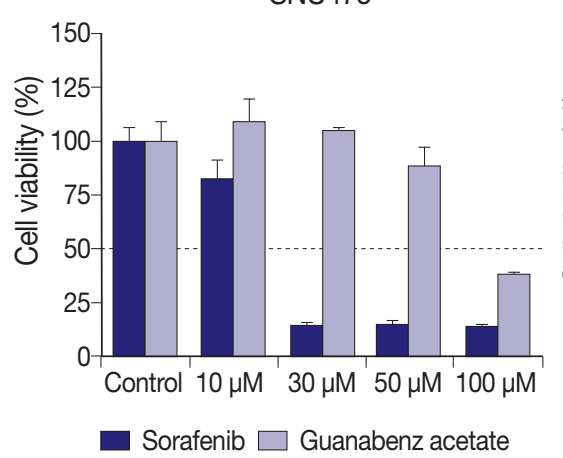

SNU423

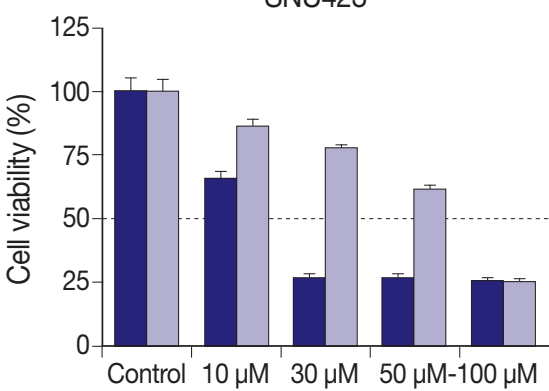

SNU398

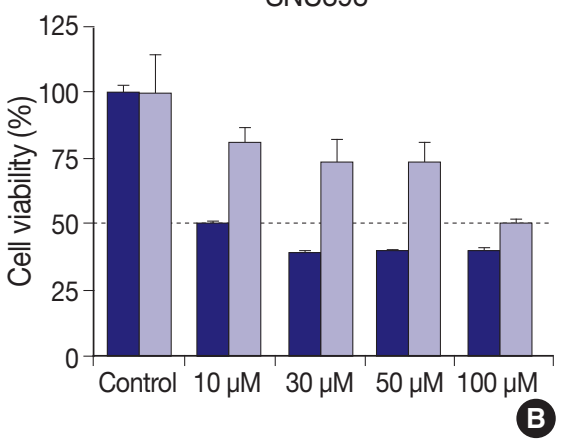

Fig. 2. (A) Drug sensitivity analysis using MTT assay of four primary cultured hepatocellular carcinoma (HCC) cells, revealing a candidate therapeutic agent for HCC. Plots show analyses of HCC cells from cases 1-4, respectively. The 41 st of 252 drugs is guanabenz acetate, which shows a significant reduction of cell proliferation $\left(\mathrm{IC}_{50}=20 \mu \mathrm{M}\right) 3$ days after drug treatment in cases 1, 2, and 4 (highlighted in red). (B) MTT assay to evaluate the inhibitory effect of guanabenz acetate and sorafenib on viability of stable HCC cell lines. Guanabenz acetate reduced the viability by more than $50 \%$ at $30 \mu \mathrm{M}$ in Hep3B cells, and at $50 \mu \mathrm{M}$ in Huh7 cells. Guanabenz acetate reduced the viability by more than $50 \%$ at $100 \mu \mathrm{M}$ in SNU423, SNU449, and SNU475 cells. Cell viability of SNU398 cells was not reduced below 50\%, even at $100 \mu \mathrm{M}$ of guanabenz acetate. Dark bars, sorafenib treatment; light bars, guanabenz acetate treatment. 


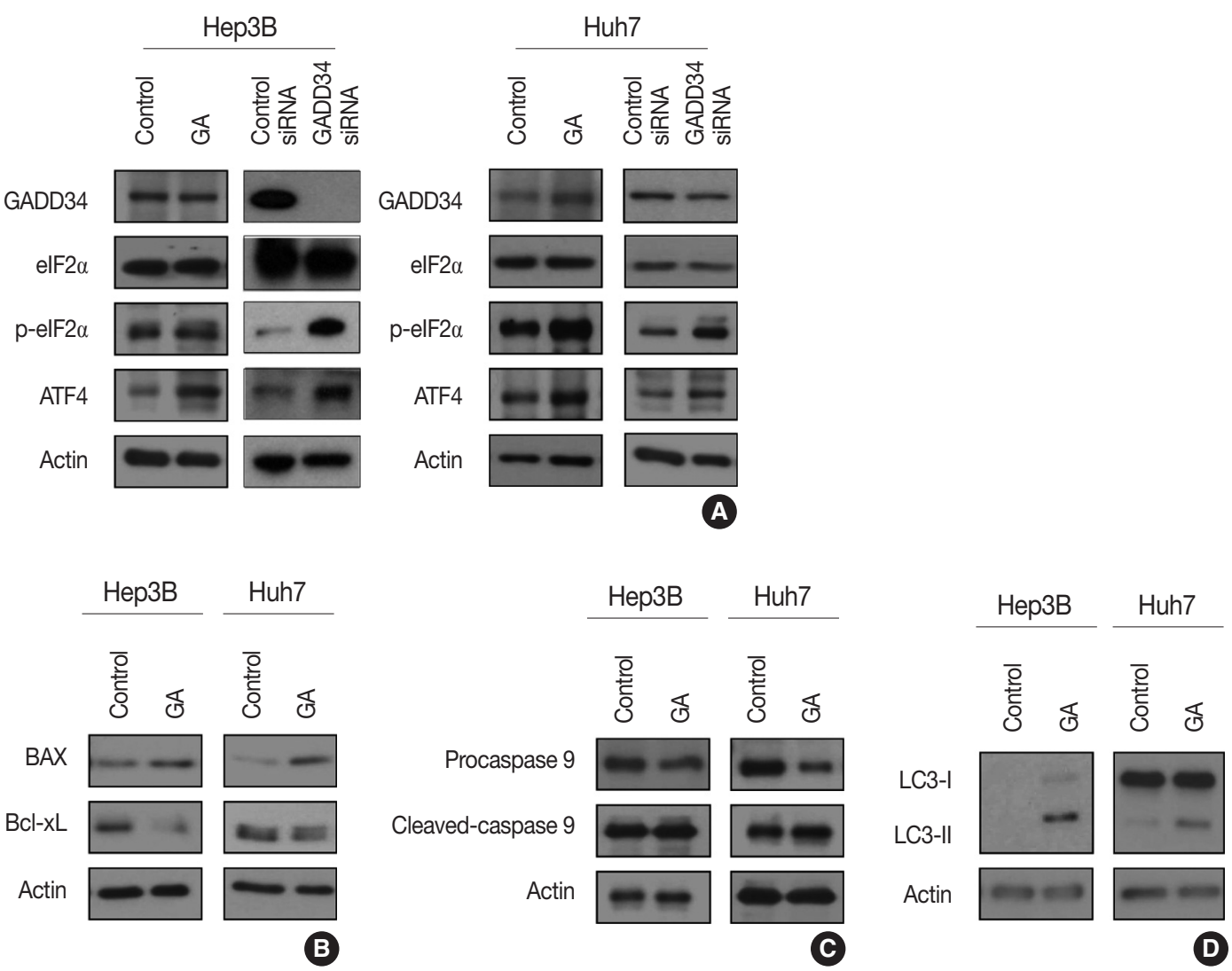

Fig. 3. (A) Western blotting showing that the effect of guanabenz acetate (GA) is associated with the protein kinase RNA-like endoplasmic reticulum kinase signaling pathway in hepatocellular carcinoma $(\mathrm{HCC})$. Similar patterns of elF2 $\alpha$ phosphorylation ( $p$-elF2 $\alpha$ ) and activating transcription factor 4 (ATF4) protein expression are observed in HCC cells treated with GA and HCC cells transfected with growth arrest and DNA damage-inducible protein 34 (GADD34) siRNA. (B-D) Western blotting showing apoptosis- and autophagy-related protein levels after GA treatment of HCC cells. (B) The protein expression levels of BAX and Bcl-xL are increased and decreased, respectively, by GA in both Hep3B and Huh7 cells. (C) The protein expression level of procaspase- 9 is decreased and that of cleaved caspase-9 is increased by GA in Hep3B and Huh7 cells. (D) The levels of autophagy-related proteins, LC3-I and LC3-II, are upregulated in GA-treated Hep3B cells, and LC3II is upregulated in GA-treated Huh 7 cells.

producing a concomitant decrease in the percentage of $\mathrm{G} 1$ phase cells (Fig. 4B). Among cell cycle-related proteins, the total protein level of p21 increased remarkably after GA treatment in Hep3B and Huh7 cells. The protein level of cyclin B1 slightly decreased, p-cdc2 remained unchanged, and cyclin E2 decreased in Hep3B cells. In Huh7 cells, however, the protein level of cyclin B1 increased, p-cdc2 remarkably decreased, and cyclin E2 slightly decreased (Fig. 4C). These results suggest that the reduced viability of HCC cells by GA treatment is regulated by cell cycle arrest. Moreover, the patterns of cell cycle arrest by GA treatment can vary among different HCC cell lines.

\section{DISCUSSION}

In this study, we discovered that GA can be repurposed as a potential anti-cancer drug for HCC patients via preclinical drug efficacy screening by using primary cultured HCC cells that we established using the surgically resected HCCs and stable HCC cell lines.

A new functional mechanism of GA was recently reported. In this mechanism, PERK activity, which is one of three pathways of the unfolded protein response (UPR) in the endoplasmic reticulum (ER), is increased by selective inactivation of GADD34mediated dephosphorylation of eIF2 $\alpha$, subsequently inducing apoptosis and autophagy (summarized in Fig. 5). ${ }^{21-24}$ UPR acts as a protective mechanism to alleviate ER stress by increasing protein-folding capacity, inhibiting general protein translation, and promoting degradation of unfolded or misfolded proteins. Interestingly, the aggregation of unfolded or misfolded proteins resulting in ER stress is associated with progression of various diseases, such as cancer, metabolic diseases, diabetes, inflammation, liver dysfunction, neurodegenerative disorders, and brain 


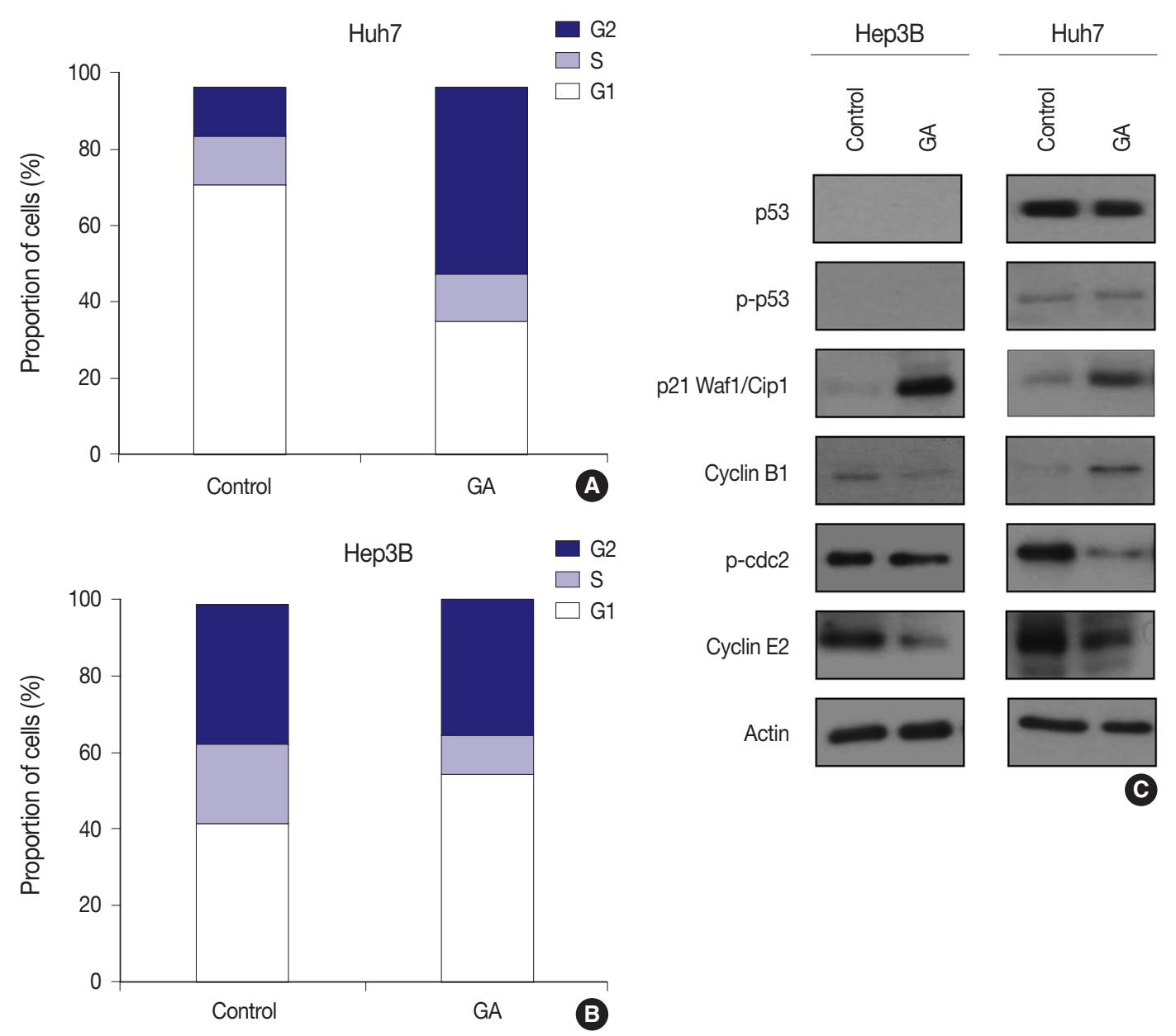

Fig. 4. Flow cytometry and western blotting showing that the type of cell cycle arrest induced by guanabenz acetate (GA) varies between hepatocellular carcinoma cell lines. (A) Flow cytometry shows increased percentage of G1 phase cells in GA-treated Hep3B cells. (B) Flow cytometry shows increased percentage of G2 phase cells in GA-treated Huh7 cells. (C) Western blotting shows the effect of GA treatment on expression levels of checkpoint proteins in the G1/S and G2/M transition and cell cycle regulating factors in Hep3B and Huh7 cells.

and heart ischemia. ${ }^{22-25}$ Moreover, when ER stress is persistent and cannot be resolved, apoptotic signaling is initiated, which eventually leads to cell death. ${ }^{23}$ Thus, the UPR signaling pathway can be an attractive target for drug discovery in diverse diseases. Recent studies have demonstrated that GA acts as a novel therapeutic agent by inhibition of dephosphorylation of eIF $2 \alpha$ in the UPR pathway for several disorders, including progressive neurodegenerative disease (especially amyotrophic lateral sclerosis), toxoplasmosis, and breast cancer. ${ }^{21,26-28}$

In the cases of HCC that were responsive to GA, the mechanisms of action were consistent with the known mechanism. First, we confirmed that GA acts on the PERK signaling pathway possibly by inactivation of GADD34 in HCC. The HCC cells treated with GA showed an increase in the phosphorylation of eIF2 $\alpha$ and expression of ATF4. Moreover, both GA-treated HCC cells and GADD34 siRNA-transfected HCC cells showed the same patterns of p-eIF2 $\alpha$ and ATF4, supporting that GA inhibits GADD34. Second, we also identified that GA reduced cell viability by both mechanisms of apoptosis and autophagy in HCC cells. In GA-treated HCC cells, apoptosis and autophagy reactions were confirmed by decreased expression of procaspase- 9 and the increased levels of cleaved caspase- 9 and LC3. We also discovered that GA independently affects the cell cycle. GA-treated HCC cells showed arrest in the G1 or G2 phase of the cell cycle, the pattern of which was different between the two HCC cell lines. Additional studies are needed to elucidate the reason why the phase of cell cycle arrest is different among HCC cell lines, and the mechanisms of these differences. In addition, the markedly upregulated p21Waf1/Cip1 protein levels in both Hep3B and Huh7 cells indicate that a p53-dependent pathway could be involved in the cell cycle arrest by GA.

The present study is the first to perform a drug repurposing 


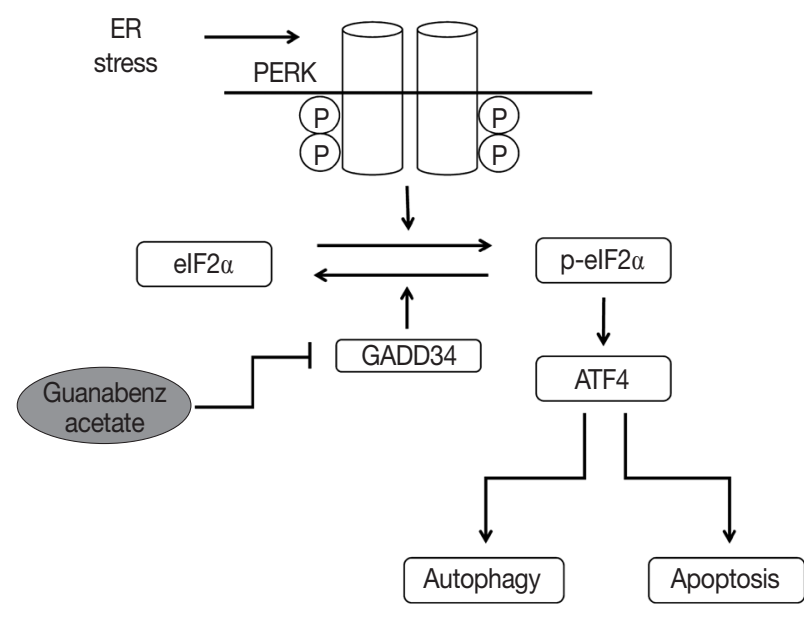

Fig. 5. Mechanisms of guanabenz acetate stimulation of the protein kinase RNA-like endoplasmic reticulum (ER) kinase (PERK) signaling pathway. Guanabenz acetate selectively inhibits growth arrest and DNA damage-inducible protein 34 (GADD34) and increases phosphorylation of eukaryotic initiation factor $2 \alpha$ (elF2 $\alpha$ ). Phosphorylation of elF2 $\alpha$ leads to increased activating transcription factor 4 (ATF4), which triggers cell apoptosis and autophagy.

study using primary cultured cells that we established from HCC patients with a high-throughput screening approach to discover novel therapeutics. The drug discovery process was developed based on genome-based drug discovery, high-throughput screening, and combinatorial chemistry. ${ }^{17}$ Contrary to expectations, novel agents identified through traditional methods have gradually declined because of high costs, time-consuming processes, and unexpected adverse reactions in clinical trials. This has led to a process called drug repositioning or repurposing. ${ }^{29,30}$ Drug repositioning is a new approach for drug discovery and is believed to offer great benefits over the traditional method of searching for new active substances, since the safety and pharmacokinetics have already been established. ${ }^{17,18,31,32} \mathrm{~A}$ representative new indication, introduced by drug repositioning, is thalidomide, a sedative hypnotic agent, which demonstrated significant clinical activity against multiple myeloma and leprosy. ${ }^{33,34}$ Moreover, the combination therapy of drugs found by drug repositioning and conventional anticancer drugs may increase efficacy and reduce adverse reactions. ${ }^{17}$ Thus, drug repositioning may become a key approach for treating variable malignancies, including HCC, in novel drug discovery.

The commercially available libraries of marketed drugs and natural compounds have been widely used in drug screening tests for a broad spectrum of diseases, including cancers, viral or fungal infections, neurodegenerative disorders, and neuromuscular disorders, but never have been used for drug efficacy tests of HCC. ${ }^{35}$ Although the unexpected effects of GA on the reduction of HCC cell viability were discovered from randomly selected drugs, the effects of GA on malignant cells have already been reported in breast cancer. A previous study revealed that combined treatment of GA and salubrinal can attenuate the malignant phenotype and tumor growth of triple negative breast cancer cells through the eIF2 $\alpha$-mediated Rac1 pathway. ${ }^{28}$ As mentioned previously, GA acts as an inhibitor of dephosphorylation of eIF2 $\alpha$ and can be used potentially as an anti-cancer drug for other malignancies, especially those originating from ER-rich organs.

This study has a few limitations. It was performed in vitro and the GA IC s0 $_{0}$ value is high for a clinically achievable dose. Therefore, additional in vitro and in vivo studies are needed to conclusively determine whether GA can be a novel anti-cancer agent for inoperable HCC patients at a clinically achievable dose. In addition, molecular and immunohistochemical studies are needed to determine the predictive marker(s) for the therapeutic effects of GA in HCC patients. Finally, the effect of GA as an anti-cancer agent could be evaluated in other malignancies of ER-rich organs.

In conclusion, $\mathrm{GA}$, which was screened in primary cultured HCC cells, reduces HCC cell viability via the combined effects of apoptosis, autophagy, and cell cycle arrest. Therefore, GA may be repositioned as an anti-cancer therapeutic agent for HCC patients.

\section{Electronic Supplementary Material}

Supplementary materials are available at Journal of Pathology and Translational Medicine (http://jpatholtm.org).

\section{ORCID}

Hyo Jeong Kang: https://orcid.org/0000-0002-5285-8282

Young-Ah Suh: https://orcid.org/0000-0002-4694-714X

Jihun Kim: https://orcid.org/0000-0002-8694-4365

Se Jin Jang: https://orcid.org/0000-0001-8239-4362

Eunsil Yu: https://orcid.org/0000-0001-5474-9744

\section{Author Contributions}

Conceptualization: HJK, HSS.

Data curation: HJK, HSS.

Formal analysis: HJK, HSS, SEL, YAS.

Methodology: HJK, HSS, SEL, YAS.

Supervision: EY, SJJ.

Visualization: HJK, HSS, YAS.

Writing—original draft: HJK.

Writing—review \& editing: EY, SJJ, JK. 


\section{Conflicts of Interest}

The authors declare that they have no potential conflicts of interest.

\section{REFERENCES}

1. Bosch FX, Ribes J, Cléries R, Díaz M. Epidemiology of hepatocellular carcinoma. Clin Liver Dis 2005; 9: 191-211.

2. Shaw JJ, Shah SA. Rising incidence and demographics of hepatocellular carcinoma in the USA: what does it mean? Expert Rev Gastroenterol Hepatol 2011; 5: 365-70.

3. Llovet JM, Burroughs A, Bruix J. Hepatocellular carcinoma. Lancet 2003; 362: 1907-17.

4. Karaman B, Battal B, Sari S, Verim S. Hepatocellular carcinoma review: current treatment, and evidence-based medicine. World J Gastroenterol 2014; 20: 18059-60.

5. Forner A, Llovet JM, Bruix J. Hepatocellular carcinoma. Lancet 2012; 379: 1245-55.

6. Venook AP, Papandreou C, Furuse J, de Guevara LL. The incidence and epidemiology of hepatocellular carcinoma: a global and regional perspective. Oncologist 2010; 15 Suppl 4: 5-13.

7. Crissien AM, Frenette C. Current management of hepatocellular carcinoma. Gastroenterol Hepatol (N Y) 2014; 10: 153-61.

8. Keating GM, Santoro A. Sorafenib: a review of its use in advanced hepatocellular carcinoma. Drugs 2009; 69: 223-40.

9. Patel A, Sun W. Molecular targeted therapy in hepatocellular carcinoma: from biology to clinical practice and future. Curr Treat Options Oncol 2014; 15: 380-94.

10. Berk V, Kaplan MA, Tonyali O, et al. Efficiency and side effects of sorafenib therapy for advanced hepatocellular carcinoma: a retrospective study by the anatolian society of medical oncology. Asian Pac J Cancer Prev 2013; 14: 7367-9.

11. De Witt Hamer PC, Van Tilborg AA, Eijk PP, et al. The genomic profile of human malignant glioma is altered early in primary cell culture and preserved in spheroids. Oncogene 2008; 27: 2091-6.

12. Seol HS, Suh YA, Ryu YJ, et al. A patient-derived xenograft mouse model generated from primary cultured cells recapitulates patient tumors phenotypically and genetically. J Cancer Res Clin Oncol 2013; 139: 1471-80.

13. Seol HS, Kang HJ, Lee SI, et al. Development and characterization of a colon PDX model that reproduces drug responsiveness and the mutation profiles of its original tumor. Cancer Lett 2014; 345: 56-64.

14. Mitra A, Mishra L, Li S. Technologies for deriving primary tumor cells for use in personalized cancer therapy. Trends Biotechnol 2013; 31:347-54.
15. Shim JS, Liu JO. Recent advances in drug repositioning for the discovery of new anticancer drugs. Int J Biol Sci 2014; 10: 654-63.

16. Würth R, Thellung S, Bajetto A, Mazzanti M, Florio T, Barbieri F. Drug-repositioning opportunities for cancer therapy: novel molecular targets for known compounds. Drug Discov Today 2016; 21: 190-9.

17. Banno K, Iida M, Yanokura M, et al. Drug repositioning for gynecologic tumors: a new therapeutic strategy for cancer. ScientificWorldJournal 2015; 2015: 341362.

18. Langedijk J, Mantel-Teeuwisse AK, Slijkerman DS, Schutjens MH. Drug repositioning and repurposing: terminology and definitions in literature. Drug Discov Today 2015; 20: 1027-34.

19. Saxena A, Becker D, Preeshagul I, Lee K, Katz E, Levy B. Therapeutic effects of repurposed therapies in non-small cell lung cancer: what is old is new again. Oncologist 2015; 20: 934-45.

20. Oprea TI, Overington JP. Computational and practical aspects of drug repositioning. Assay Drug Dev Technol 2015; 13: 299-306.

21. Wang L, Popko B, Tixier E, Roos RP. Guanabenz, which enhances the unfolded protein response, ameliorates mutant SOD1-induced amyotrophic lateral sclerosis. Neurobiol Dis 2014; 71: 317-24.

22. Rozpedek W, Markiewicz L, Diehl JA, Pytel D, Majsterek I. Unfolded protein response and PERK kinase as a new therapeutic target in the pathogenesis of Alzheimer's disease. Curr Med Chem 2015; 22: 3169-84.

23. Shah SZ, Zhao D, Khan SH, Yang L. Unfolded protein response pathways in neurodegenerative diseases. J Mol Neurosci 2015; 57: 529-37.

24. Stone $\mathrm{S}$, Lin $\mathrm{W}$. The unfolded protein response in multiple sclerosis. Front Neurosci 2015; 9: 264.

25. Harada M, Nose E, Takahashi N, et al. Evidence of the activation of unfolded protein response in granulosa and cumulus cells during follicular growth and maturation. Gynecol Endocrinol 2015; 31: 783-7.

26. Vieira FG, Ping Q, Moreno AJ, et al. Guanabenz treatment accelerates disease in a mutant SOD1 mouse model of ALS. PLoS One 2015; 10: e0135570.

27. Benmerzouga I, Checkley LA, Ferdig MT, Arrizabalaga G, Wek $\mathrm{RC}$, Sullivan WJ Jr. Guanabenz repurposed as an antiparasitic with activity against acute and latent toxoplasmosis. Antimicrob Agents Chemother 2015; 59: 6939-45.

28. Hamamura K, Minami K, Tanjung N, et al. Attenuation of malignant phenotypes of breast cancer cells through eIF2alpha-mediated downregulation of Rac1 signaling. Int J Oncol 2014; 44: 1980-8.

29. Jadamba E, Shin M. A systematic framework for drug repositioning from integrated omics and drug phenotype profiles using pathway-drug network. Biomed Res Int 2016; 2016: 7147039. 
30. Ashburn TT, Thor KB. Drug repositioning: identifying and developing new uses for existing drugs. Nat Rev Drug Discov 2004; 3 : 673-83.

31. Temesi G, Bolgár B, Arany A, Szalai C, Antal P, Mátyus P. Early repositioning through compound set enrichment analysis: a knowledgerecycling strategy. Future Med Chem 2014; 6: 563-75.

32. Mizushima T. Identification of a molecular mechanism for actions of existing medicines and its application for drug development. Yakugaku Zasshi 2012; 132: 713-20.
33. Schwab C, Jagannath S. The role of thalidomide in multiple myeloma. Clin Lymphoma Myeloma 2006; 7: 26-9.

34. Walker SL, Waters MF, Lockwood DN. The role of thalidomide in the management of erythema nodosum leprosum. Lepr Rev 2007; 78: 197-215.

35. Choulier L, Nomine Y, Zeder-Lutz G, et al. Chemical library screening using a SPR-based inhibition in solution assay: simulations and experimental validation. Anal Chem 2013; 85: 8787-95. 